\title{
Pre- and postoperative headache in patients with meningioma
}

\author{
Franziska Guenther', Ferdinand Swozil' ${ }^{2}$, Stefan Heber ${ }^{3}$, \\ Michael Buchfelder ${ }^{2}$, Karl Messlinger' and \\ Michael JM Fischer ${ }^{1,3}$ (1)
}

\begin{abstract}
Background: Meningiomas are generally slowly growing intracranial tumors. They are often incidentally diagnosed, given that symptoms may be absent even in cases of an enormous tumor size. Headache is a frequent but not consistent symptom. Therefore, we examined the association between structural, biochemical and histochemical tumor parameters with preoperative as well as postoperative occurrence of headache.

Methods: In our study, we prospectively investigated 69 consecutive patients enrolled for meningioma neurosurgery. Anatomical, histological and biochemical parameters were acquired, and headache parameters were registered from the clinical report and from a questionnaire filled by the patients before neurosurgery. The headache was re-evaluated one year after neurosurgery. The study was designed to exploratively investigate whether there is an association of acquired clinical and biological parameters with the occurrence of preoperative and postoperative headache.

Results: Edema diameter and the proliferation marker MIB-I were negatively associated with the incidence and intensity of preoperative headache, while the content of prostaglandin $E_{2}$ in the tumor tissue was positively associated with preoperative headache intensity. Headache was more prevalent when the meningioma was located in the area supplied by the ophthalmic trigeminal branch. Compared to preoperative headache levels, an overall reduction was observed one year postoperative, and patients with a larger tumor had a higher headache remission. In parietal and occipital meningiomas and in those with a larger edema, the percentage of the headache remission rate was higher compared to other locations or smaller edema. Multivariable analyses showed an involvement of substance $P$ and prostaglandin $\mathrm{E}_{2}$ in preoperative headache.

Conclusions: The study demonstrates new associations between meningiomas and headache. The postoperative headache outcome in the presented patient sample is encouraging for the performed neurosurgical intervention. These results should be tested in a prospective study that incorporates all patients with meningiomas.
\end{abstract}

\section{Keywords}

Meningioma, neurosurgery, preoperative headache, postoperative headache

Date received: 2I December 2017; revised: 13 March 2018; 20 April 2018; 25 May 2018; accepted: 29 May 2018

\author{
Abbreviations \\ CGRP calcitonin gene-related peptide \\ NO nitric oxide \\ $\mathrm{PGE}_{2}$ prostaglandin E2
}

\section{Introduction}

Meningiomas are the most frequent type of all primary intracranial tumors. In more than $90 \%$ of cases they are
'Institute of Physiology and Pathophysiology, University of ErlangenNürnberg, Erlangen, Germany

${ }^{2}$ Department of Neurosurgery, University of Erlangen-Nürnberg, Erlangen, Germany

${ }^{3}$ Center for Physiology and Pharmacology, Medical University of Vienna, Vienna, Austria

\section{Corresponding author:}

Karl Messlinger, Institute of Physiology and Pathophysiology, University of Erlangen-Nürnberg, Universitaetsstrasse 17, 91054 Erlangen, Germany. Email: karl.messlinger@fau.de 
benign (1) and are clinically only recognized when they are accompanied by neurological deficits or abnormal functions. Meningiomas generally grow slowly and remain frequently untreated until serious symptoms occur. The aim of treatment is the complete surgical removal of the tumor, which may be difficult or impossible due to the anatomical location (2). Headache is the most frequent symptom and occurs in about two thirds of the patients $(3,4)$. Whether a meningioma causes a headache may depend on compression of specific structures or an increase in intracranial pressure. Other reasons may be endogenously formed substances that are assumed to interact with intracranial nociception. Among such substances, which may be elevated in patients' plasma during headaches or which can induce headaches, are neuropeptides, in particular calcitonin gene-related peptide (CGRP), nitric oxide (NO) and its metabolites, and prostaglandin $\mathrm{E}_{2}$ (PGE2). In a histochemical investigation of pituitary tumors, substance $\mathrm{P}$ or CGRP immunoreactivity was found in about one quarter of specimens; however, this was not correlated with the occurrence of headache $(5,6)$. Also, no correlation was found between gene expression of the meningioma subtypes and particular features such as vasodilation, associated headache or key substances like neuropeptides or NO synthases (7). In highly malignant glioblastomas, the neuronal NO synthase isoform was increased but this was not the case in lower-grade glioblastomas or meningiomas (8). The prostaglandin $\mathrm{PGE}_{2}$ is a common inflammatory mediator, a metabolite of arachidonic acid formed through cyclooxygenases 1 and 2. In addition to its vasodilatory effect, $\mathrm{PGE}_{2}$ sensitizes nociceptors (9) and also has central nociceptive effects (10). Cyclooxygenase inhibitors are among the most frequently used drugs for unspecific headaches. The inducible cyclooxygenase 2 was demonstrated immunohistochemically in many meningiomas and, as for the NO synthase, the degree of cyclooxygenase 2 expression was associated with the malignancy of the tumors (11). However, meningioma-associated headaches did not correlate with cyclooxygenase 2 expression (7). The growth of meningiomas seems to be promoted in an autocrine manner (12), but also by the peptide cholecystokinin. A possible link between the production of cholecystokinin and headache has not been studied so far. The first aim of our study was to identify biological meningioma parameters that are associated with preoperative occurrence and intensity of headache. Prior studies looked at postoperative outcome other than headache in patients with meningioma, including investigation of the meningioma location, the meningioma cell type and grading, coexisting symptoms, an occurrence of particular postoperative symptoms, the operating techniques, age subpopulations, the recurrence rate and the outcome due to additional procedures or diagnostics. $(7,13-24)$. Thus, the second aim of our study was to identify parameters in an operated patient sample that are predictive for postoperative headache, which has not been focused on before.

\section{Materials and methods}

\section{Ethical approval and patient recruitment}

The study was approved by the ethical review board of the Medical Faculty of the University of ErlangenNürnberg. In total, all 69 patients diagnosed with meningioma and operated on at the Department of Neurosurgery of the University of Erlangen-Nürnberg in the period 2010-2012 were included in the study, no patients were excluded. A written informed consent was provided by all patients or their legal representatives. Patient charts containing clinical data, laboratory measurements and radiological results of the patients were acquired for analysis.

\section{Questionnaire and data acquisition}

Within the 69 included operated patients, 59 completed a headache-focused questionnaire developed by Dr. Michael Küster (KSFB-Bonn, devised by the Pain Center of the German Pain Society in Bonn, Germany). The original German questionnaire is available with permission as a supplement, in addition there is also an English translation of the questionnaire. The patients answered the questionnaire at least until the day before the surgery. In all cases, the questionnaire was completed before an optional medication with dexamethasone. Some patients had help from relatives to complete the questionnaire. From this questionnaire with 39 items, only the following items were selected to investigate a possible association with headache and biological parameters of the meningioma. Patients with more than infrequent headache (once per month, according to ICHD 3-beta) were classified as headache-positive and asked to rate the typical intensity of their headache. The headache intensity was rated on a numerical rating scale ranging from 0 to 51. The numerical rating scale was additionally graded by a verbal pain rating, the latter not being considered in our analysis. None of the patients with meningioma undergoing neurosurgery in the study period were excluded; three of these patients were considered to have meningiomatosis. Within the patient cohort, the prevalence of other diseases was recorded. Three patients had an intracranial pathology, that is, one patient had a cerebral infarction, one had a thalamus infarction, and one had a 
prolactinoma. In addition, one patient suffered from epilepsy, two had a psychoorganic syndrome, one had leukemia, one had Cushing's disease, one had multiple sclerosis, and one had a spinal osteoblastoma. One patient suffered from migraine, which persisted after the surgery. In addition, the localization of the headache and the intake of medication for pain relief was recorded.

In patients with preoperative headache, this was classified as "7.4.1 Headache attributed to intracranial neoplasm" according to ICHD 3-beta (25). This is due to the presence of headache, an intracranial neoplasia, the temporal relation of headache to the intracranial neoplasia in most of the patients and headache relapse in relation to the neurosurgery. For those patients in which 7.4.1 $\mathrm{C} 2 \mathrm{~b}$ "headache has significantly improved in temporal relation" to the operation does not apply, the best classification might be 7.4.1D, that is patients "not better accounted for by another ICHD3 diagnosis".

The occurrence of postoperative headaches one year after surgery was assessed during a follow up visit or via phone contact by the neurosurgeon. The postoperative evaluation determined headache with the same criteria and on the same $0-51$ pain scale as used by the preoperative questionnaire. The meningioma size was determined in preoperative magnetic resonance imaging. The diameter of the preoperative edema was determined by the neurosurgeon F.S. using preoperative magnetic resonance imaging.

\section{Biological tumor analysis}

Samples of 61 meningiomas were processed for immunohistochemistry and their neuropeptide content. The resected tissue was placed in isotonic electrolyte solution and immediately frozen in liquid nitrogen until further processing. For immunohistochemistry, one part was fixed in $4 \%$ paraformaldehyde with $0.01 \%$ picric acid. The fixation period was adjusted for the size of the tissue, assuming a diffusion of about $0.5 \mathrm{~mm} / \mathrm{h}$. The samples were incubated in phosphate buffered saline overnight and immersed in $10 \%$ sucrose for 24 hours before freezing. Finally, they were embedded in TissueTek, frozen in methylbutane and stored at $-20^{\circ} \mathrm{C}$. The second part of the tumor sample was stored at $-20^{\circ} \mathrm{C}$ without fixation to determine the total content of substances addressed below (26). Briefly, the cryoconserved samples were thawed and washed in synthetic interstitial fluid containing, in mM: $107.8 \mathrm{NaCl}, 26.2 \mathrm{NaCO}_{3}, 9.64 \mathrm{Na}$-gluconate, 7.6 sucrose, 5.55 glucose, $3.48 \mathrm{KCl}, 1.67 \mathrm{NaH}_{2} \mathrm{PO}_{4}, 1.53$ $\mathrm{CaCl}_{2}$ and $0.69 \mathrm{MgSO}_{4}$ (27). After weighting, samples were immersed in $2 \mathrm{M}$ acetic acid, heated to $95^{\circ} \mathrm{C}$ and boiled for 10 minutes. Afterwards, the tissues were homogenized with a custom-made homogenizer. The samples were boiled a second time for 10 minutes at $95^{\circ} \mathrm{C}$ in $2 \mathrm{M}$ acetic acid and centrifuged for 30 minutes at $10,000 \mathrm{~g}$. The supernatant was removed and $3 \mathrm{M}$ $\mathrm{NaOH}$ was added to adjust to a $\mathrm{pH}$ of 5.2-7.4. In these samples, the content of $\mathrm{PGE}_{2}$, substance $\mathrm{P}$ and CGRP was determined by specific enzyme-linked immune assays as indicated by the manufacturer (Bertin, France) and as previously described (28). Whole protein content was determined by a kit (ThermoFischer, Waltham, MA). The protein concentration and the neuropeptide content of the samples was detected photometrically by a microplate reader (Opsys MR-Dynex Technologies, Germany). The tumor proliferation index MIB-1 of most tumors has been described (29). This uses a monoclonal antibody as a cell proliferation marker on routine histologic material to determine the proliferative activity of Ki-67 protein (30). The latter is present during all active phases of the cell cycle and is therefore a useful tool for detecting the clinical course of the disease.

\section{Statistical procedures}

The study was prospective and designed to investigate potential associations of acquired parameters with occurrence of preoperative and postoperative headache in an exploratory manner. Further, associations between acquired parameters had an exploratory character and therefore no endpoints were predefined. First, independent parameters were pairwise tested for correlation. This was calculated with a product-momentum correlation for two continuous variables and by a Spearman correlation when at least one non-parametric variable was included. Due to the exploratory nature of the present study, in this phase no correction for multiple testing was employed. Chi-square tests were used to test $2 \times 2$ and $2 \times 3$ case contingency tables with a Yates's correction where required. Two groups were compared by t-tests for independent samples. Bonferroni-Holm correction was used to adjust $p$-levels in case of linked tests. Multiple groups were evaluated by analysis of variance (ANOVA), followed by Tukey's HSD post-hoc test for pairwise comparisons.

To specifically address a possible involvement of the neuropeptides CGRP and substance $\mathrm{P}$ and the lipid mediator $\mathrm{PGE}_{2}$ in preoperative headache, two hierarchical, multiple regression models were applied: To predict the occurrence (yes and no) of preoperative headache in all patients, binary logistic regression was used. Firth logistic regression was applied due to the low number of patients without preoperative headache taking pain medication $(31,32)$. For patients with preoperative headache, its intensity (1-51) was modeled using multiple linear regression. In both logistic and 
linear regression, the following combination of predictors was used: Model 1 contained pain medication (yes and no) and maximal tumor diameter; in Model 2, concentrations of CGRP, Substance $\mathrm{P}$ and $\mathrm{PGE}_{2}$, all $\log$-transformed to base 2, were entered in addition. The likelihood ratio Chi-square statistic was used to test i) if Model 1 predicts headache occurrence and intensity; ii) if Model 2 predicts headache occurrence and intensity; iii) if Model 2 predicts headache occurrence and intensity better than Model 1; iv) to what extent the individual variables contribute to the models; and v) if the individual variables predict headache occurrence and its intensity.

All statistical tests were two-sided, $p<0.05$ was considered statistically significant. Data are presented as mean \pm SEM, in case of skewed distributions with a Shapiro-Wilk test for normality of $p<0.05$, median and interquartile range is given. Analyses were performed using Statistica (StatSoft, Tulsa, USA) and IBM SPSS Statistics 24 .

Table I. Patient characteristics and sample size in absolute numbers and percentage.

\begin{tabular}{|c|c|c|}
\hline & Count & $\%$ \\
\hline $\begin{array}{l}\text { Patients included (Meningioma diagnosis } \\
\text { and respective neurosurgery) }\end{array}$ & 69 & 100 \\
\hline Female & 47 & 68 \\
\hline Male & 22 & 32 \\
\hline $\begin{array}{l}\text { Preoperative magnetic resonance imagi- } \\
\text { ng (includes meningioma measurement } \\
\text { and location) }\end{array}$ & 69 & 100 \\
\hline $\begin{array}{l}\text { Preoperative headache assessed (ques- } \\
\text { tionnaire completed) }\end{array}$ & 62 & 90 \\
\hline Without preoperative headache & 24 & 35 \\
\hline With preoperative headache & 38 & 55 \\
\hline Postoperative headache assessed & 65 & 94 \\
\hline Without postsurgical headache & 53 & 77 \\
\hline With postsurgical headache & 12 & 17 \\
\hline Pre- and postoperative headache assessed & 60 & 87 \\
\hline Without preoperative headache & 23 & 33 \\
\hline With preoperative headache & 37 & 54 \\
\hline $\begin{array}{l}\text { With preoperative, without postsurgical } \\
\text { headache }\end{array}$ & 28 & 41 \\
\hline $\begin{array}{l}\text { With preoperative, with postsurgical } \\
\text { headache }\end{array}$ & 9 & 13 \\
\hline \multicolumn{3}{|l|}{ Biological parameters } \\
\hline Grading (histopathology) & 68 & 99 \\
\hline MIB-I (histopathology) & 24 & 35 \\
\hline Neuropeptide measurement & 59 & 86 \\
\hline
\end{tabular}

\section{Results}

Meningiomas of 69 patients ( 47 female) were removed by neurosurgery (Table 1 ). There was no significant age difference between the sexes $(p=0.65$, U-test $)$, the median age of all patients was 58 (range 34-82) years. However, the meningioma grade was higher and the maximum diameter and surrounding edema was larger in males compared to females $(p=0.046$, Chi-square, 0.035 and 0.040, U-tests, Figure S1). Preoperative questionnaires and clinical data were investigated, primarily for their association with preand post-operative headache. The largest diameter, which was used for classification of the meningiomas, was $39 \pm 2 \mathrm{~mm}$ on average; in 17 tumors it was below $3 \mathrm{~cm}$, in 42 within the range of [3-6] $\mathrm{cm}$, and in 10 above $6 \mathrm{~cm}$. More than half $(36 / 69)$ of the meningiomas were localized in the frontal lobe, five temporal, 16 parietal and 12 occipital. The edema around the meningioma on preoperative MR images had a median diameter of $10 \mathrm{~mm}$ (interquartile range $0-35 \mathrm{~mm}$ ). The edema diameter was correlated with the measures of tumor size, including largest diameter and tumor volume $(\mathrm{R}=0.51,0.40$ and all $p<0.001$, productmomentum correlation, Figure S2(a)-(c)). The edema was smaller for meningiomas in the occipital lobe and for meningiomas in areas innervated by $\mathrm{C} 1-\mathrm{C} 3 \mathrm{com}-$ pared to other meningiomas $(p=0.003$ and 0.047 , Utest, Figure S2(d)). The median substance P levels of the meningioma tissue were $29 \mathrm{pg} / \mathrm{ml}$ (interquartile range 19-55), and higher values were observed in tumors with larger volume $(\mathrm{R}=0.31, p=0.035$, productmomentum correlation, Figure S3(a)). Compared to the other lobes, frontal meningiomas had higher substance $\mathrm{P}$ levels $(p=0.047$, bootstrapped t-test, Figure S3(b)). Median CGRP levels of the meningioma tissue were $6 \mathrm{pg} / \mathrm{ml}$ (interquartile range 2-10) and were not associated with tumor parameters or headache occurrence. Dexamethasone was given in 30 of 69 patients, based on the edema in the magnetic resonance imaging. Dexamethasone application to reduce volume for the operation was more frequent in tumors with higher WHO grading and larger maximum diameter ( $p=0.002$ and $p=0.002$, U-tests). Based on these results, unsurprisingly patients receiving glucocorticoids were predominantly male ( $p=0.021$, Chi-square).

\section{Preoperative headache: Univariate analyses}

Preoperative headache exceeding an infrequent rate (once per month, according to ICHD 3-beta) was reported by $60 \%(38 / 63)$ of patients. The headaches did not conform to characteristic primary headaches such as migraine or cluster headache, no characteristic pattern of the headache was recorded, as it was reported in previous studies (33). The preoperative 


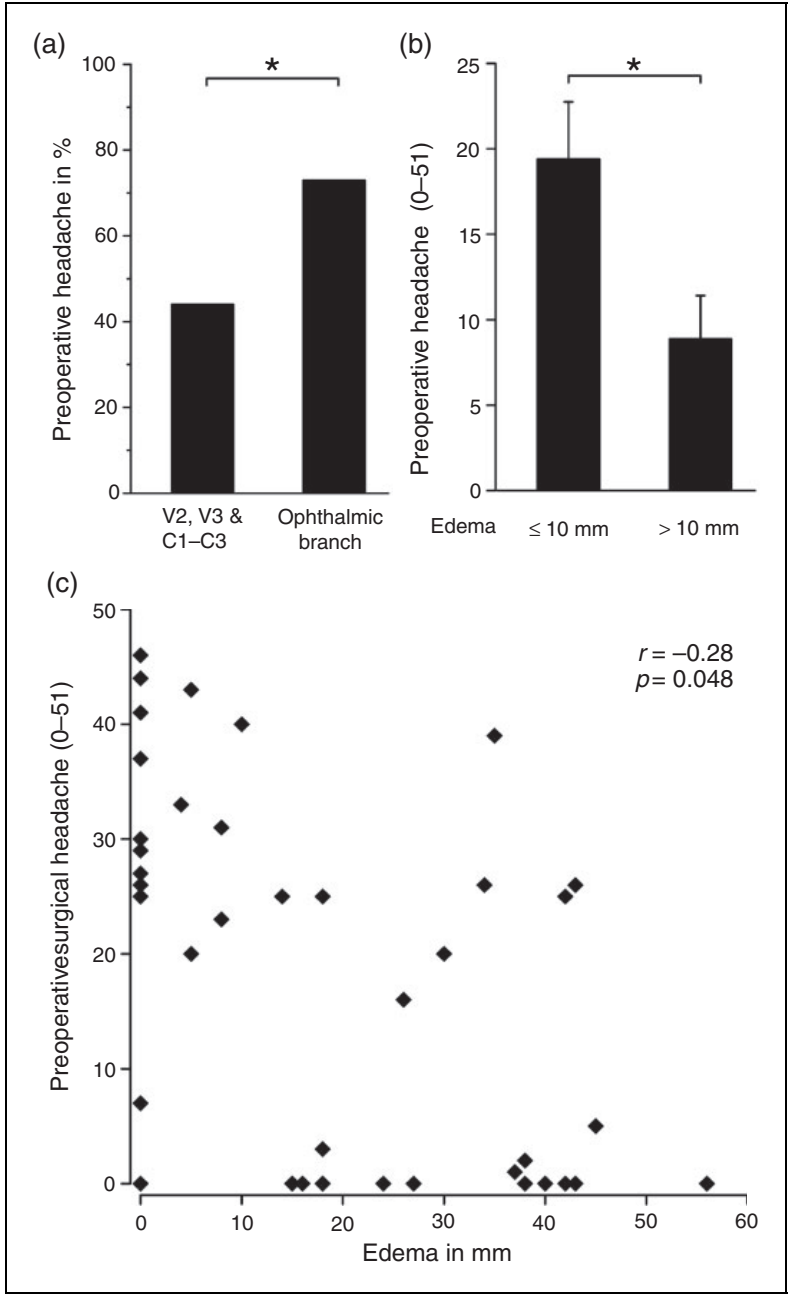

Figure I. (a) Preoperative headache is associated with innervation. Meningiomas were classified according to the innervation territory of the three trigeminal branches and spinal nerves $\mathrm{Cl}-$ C3. Meningiomas in the territory of the ophthalmic branch were more frequently associated with headache compared to the other cases. (b) Preoperative edema diameter, here thresholded at $10 \mathrm{~mm}$, is negatively correlated with the preoperative headache rating. (c) Scatterplot of the edema around the tumors and the preoperative headache intensity.

incidence of headache was $71 \%(27 / 38)$ in patients with meningiomas innervated by the first trigeminal branch, which is higher than $44 \%(11 / 25)$ for the second and third trigeminal branch $(p=0.032$, Chi-square test, Figure 1). Compared to the functional classification, in a simpler anatomical classification to frontal, temporal, parietal and occipital, no significant differences were observed. The preoperative incidence or intensity of the headache was not associated with the maximum tumor diameter $\left(\mathrm{R}_{\mathrm{s}}=0.004\right.$ and $-0.076, p=0.98$ and 0.57 , Spearman correlations). The location of the sensation of headaches was not associated with the location of meningiomas. This is in agreement with a previous intraoperative study, where stimulation of
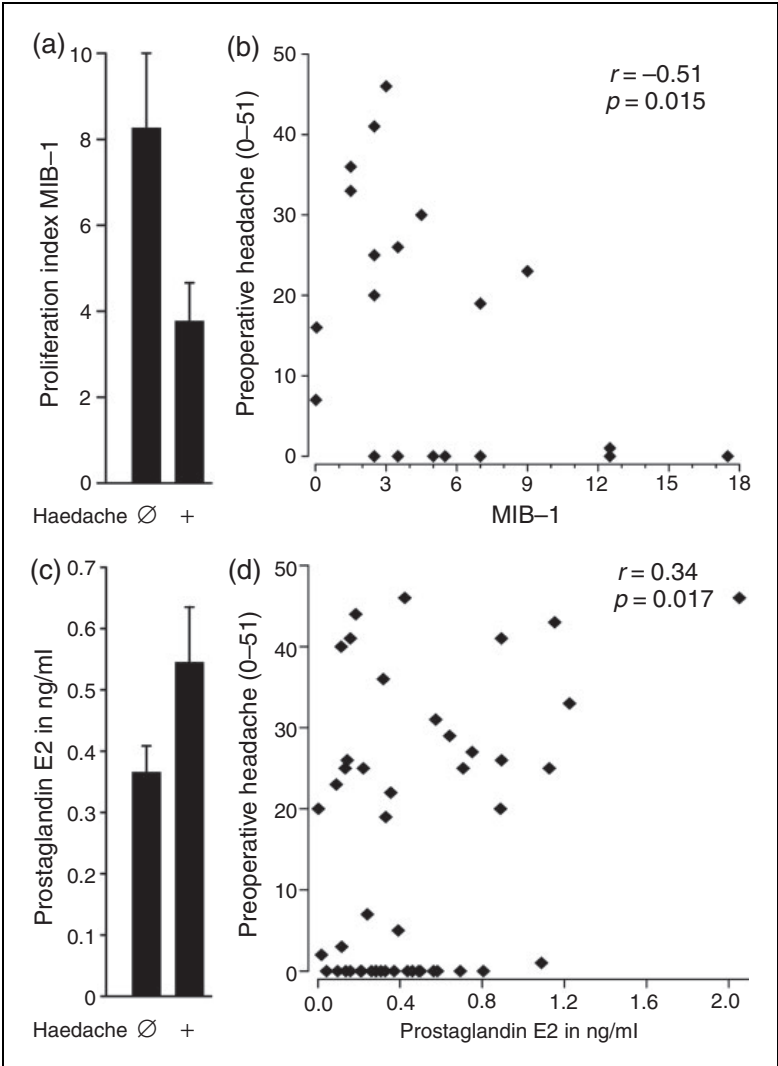

Figure 2. Biological parameters associated with preoperative headache. (a) Patients without preoperative headache had a higher tumor proliferation index MIB-I compared to patients without preoperative headache. (b) The MIB-I was negatively correlated with the intensity of preoperative headache. (c) Prostaglandin E2 concentration in meningiomas of patients with and without preoperative headache. (d) The levels of prostaglandin E2 in the meningioma were correlated with the intensity of preoperative headache.

different sites of the exposed dura mater caused widespread referral of pain, which did not coincide with the expected trigeminal areas (34). For the 12 patients, who had both a unilateral headache and a unilateral meningioma, there was no positive or negative association between the side of the headache and the side of the meningioma ( $p=0.61$, Chi-square test). Patients with an edema above $10 \mathrm{~mm}$ had a preoperative headache rating of $9 \pm 3$, which is lower than the $19 \pm 3$ reported by patients with smaller edema $(p=0.044, \mathrm{n}=24$ and 28, U-test, Figure 1(b)). The edema around the tumors was negatively associated with the preoperative headache intensity $(\mathrm{R}=-0.28, p=0.048$, product-momentum correlation, Figure 1(c)). The tumor proliferation index MIB-1 was higher in patients without than in patients with preoperative headache $(p=0.020$, U-test, determined in 24 patients, Figure 2(a)). The MIB-1 was negatively associated with the intensity of preoperative headache $(\mathrm{R}=-0.51, p=0.015$, product-momentum 
Table 2. Hierarchical multivariable regression models testing the effects of CGRP, substance $P$ and $P G E_{2}$ concentrations within meningiomas on preoperative headache occurrence and intensity.

\begin{tabular}{|c|c|c|c|c|c|c|c|c|c|c|c|c|}
\hline & \multicolumn{5}{|c|}{ Firth logistic regression } & \multicolumn{5}{|c|}{ Linear regression } & \multicolumn{2}{|l|}{ Overall } \\
\hline & \multicolumn{3}{|c|}{$95 \% \mathrm{Cl}$ for Odds Ratio } & \multirow[b]{2}{*}{$\chi^{2}(\mathrm{df})$} & \multirow[b]{2}{*}{$p$} & \multicolumn{3}{|c|}{$95 \% \mathrm{Cl}$ for $\mathrm{b}$} & \multirow[b]{2}{*}{$\chi^{2}(d f)$} & \multirow[b]{2}{*}{$p$} & \multirow[b]{2}{*}{$\chi^{2}(\mathrm{df})$} & \multirow[b]{2}{*}{$p$} \\
\hline & Lower & $\begin{array}{l}\text { Odds } \\
\text { Ratio }\end{array}$ & Upper & & & Lower & $b$ & Upper & & & & \\
\hline Model I & & & & $13.0(2)$ & 0.002 & & & & $4.72(2)$ & 0.09 & & \\
\hline Pain medication (yes/no) & 3.33 & 17.9 & 186 & $12.8(1)$ & $<0.001$ & 2.51 & 8.41 & 19.3 & $2.36(1)$ & 0.13 & $15.2(2)$ & $<0.001$ \\
\hline Maximal tumor diameter $(\mathrm{cm})$ & 0.60 & 1.08 & 1.95 & $0.06(1)$ & 0.80 & -5.81 & -2.58 & 0.66 & $2.5 I(I)$ & 0.11 & $2.57(2)$ & 0.28 \\
\hline Model 2 & & & & $12.4(5)$ & 0.029 & & & & $15.9(5)$ & 0.007 & & \\
\hline Pain medication (yes/no) & 2.56 & 12.7 & 122 & $10.5(1)$ & 0.001 & 1.18 & 9.72 & 18.3 & $4.86(1)$ & 0.028 & $15.4(2)$ & $<0.001$ \\
\hline Maximal tumor diameter $(\mathrm{cm})$ & 0.63 & 1.12 & 2.08 & $0.15(1)$ & 0.70 & -6.93 & -4.00 & -1.08 & $6.73(1)$ & 0.009 & $6.88(2)$ & 0.032 \\
\hline CGRP (log2 pg/ml) & 0.41 & 0.80 & 1.50 & $0.47(\mathrm{I})$ & 0.49 & -1.61 & 1.28 & 4.17 & $0.8 \mathrm{I}(\mathrm{I})$ & 0.37 & $1.28(2)$ & 0.52 \\
\hline Substance $P(\log 2 \mathrm{pg} / \mathrm{ml})$ & 0.57 & 1.08 & 2.21 & $0.06(1)$ & 0.81 & 1.43 & 4.42 & 7.41 & $7.65(1)$ & 0.006 & $7.70(2)$ & 0.021 \\
\hline Prostaglandin $\mathrm{E}_{2}(\log 2 \mathrm{pg} / \mathrm{ml})$ & 0.69 & 1.14 & 1.77 & $0.39(1)$ & 0.53 & 0.55 & 2.49 & 4.42 & $6.04(1)$ & 0.014 & $6.43(2)$ & 0.040 \\
\hline Comparison of Model I and 2 & & & & $0.53(3)$ & 0.91 & & & & $11.2(3)$ & 0.010 & & \\
\hline
\end{tabular}

Note. Bold numbers denote significant associations with preoperative headache.

correlation, Figure 2(b)). Median $\mathrm{PGE}_{2}$ levels in meningioma tissue were $354 \mathrm{pg} / \mathrm{ml}$ (interquartile range 158$581)$ and were positively correlated with the intensity of preoperative headache $(\mathrm{R}=0.34, p=0.019, \mathrm{n}=48$, product-momentum correlation, Figure 2(c),(d)). Substance $\mathrm{P}$ levels failed to reach significance $(\mathrm{R}=0.37, p=0.071$, $\mathrm{n}=44$, product-momentum correlation).

\section{Preoperative headache: Multivariable analyses}

Multivariable analyses were used to assess to what degree CGRP, Substance $\mathrm{P}$ and $\mathrm{PGE}_{2}$ predict occurrence and intensity of preoperative headache while controlling for some known confounders. For occurrence of preoperative headache, Firth logistic regression was used for its intensity linear regression. Results are listed in Table 2. A total of 36 patients took no medication. The sample size was insufficient to allow a sub-analysis for individual substances or substance classes with adequate statistical power (10 patients took acetyl salicylic acid, 10 ibuprofen, four metamizol, three acetyl salicylic acid + paracetamol + caffeine, two diclophenac, one amitryptilin, one etoricoxib, one paracetamol, one tilidine, one tramadol and two patients a triptan).

In Firth logistic regression, Model 1 including the variables pain medication and maximal tumor diameter predicted the occurrence of headache significantly better than a basic model without predictors (Model 1: $\left.\chi^{2}(2)=13.0, p=0.002\right)$, whereby only the variable pain medication contributed significantly $\left(\chi^{2}(1)=12.8\right.$, $p<0.001)$. To test the predictive value of CGRP, substance $\mathrm{P}$ and $\mathrm{PGE}_{2}$, their concentration was added in addition (Model 2). Although Model 2 also significantly predicted headache occurrence in general
(Model 2: $\left.\chi^{2}(5)=12.4, p=0.029\right)$, it was not better than Model 1 (Model 2 vs. Model 1, $p=0.91$ ).

In multiple linear regression, Model 1 did not significantly predict headache intensity $\left(\mathrm{R}^{2}=0.19\right.$, $\left.\chi^{2}(2)=4.28, p=0.09\right)$. Addition of CGRP, substance $\mathrm{P}$ and $\mathrm{PGE}_{2}$ concentration significantly improved the model, increasing the coefficient of determination by 0.36 to 0.55 , which was statistically significant (Model 2 vs. Model 1: $\left.\chi^{2}(3)=11.2, p=0.010\right)$. While the effect of CGRP was not significant, substance $\mathrm{P}$ and $\mathrm{PGE}_{2}$ significantly predicted headache intensity $(p=0.006$ and 0.014 ). The partial correlation coefficients for substance $\mathrm{P}$ and $\mathrm{PGE}_{2}$ concentration with preoperative headache were 0.512 and 0.509 , respectively.

Calculation of overall likelihood ratio statistics with respective $p$-values showed that pain medication, maximal tumor diameter, substance $\mathrm{P}$ and $\mathrm{PGE}_{2}$ concentration significantly predicted preoperative headache, whereas no such effect was observed for CGRP concentration.

\section{Post-operative headache}

The percentage of all patients without postoperative headache increased with the T-staging $\left(\mathrm{R}_{\mathrm{s}}=0.25\right.$, $p=0.043$, Spearman correlation, Figure 3(a)). Also, within patients with preoperative headache, the percentage of patients who became headache-free after the surgery increased with the T-staging $\left(\mathrm{R}_{\mathrm{s}}=0.34, p=0.042\right.$, Spearman correlation, Figure S4). These results are similar when an at least $\geq 50 \%$ reduction in the postoperative headache rating is considered instead of complete absence of headache. Further of interest is the predictive value of the anatomical location. 


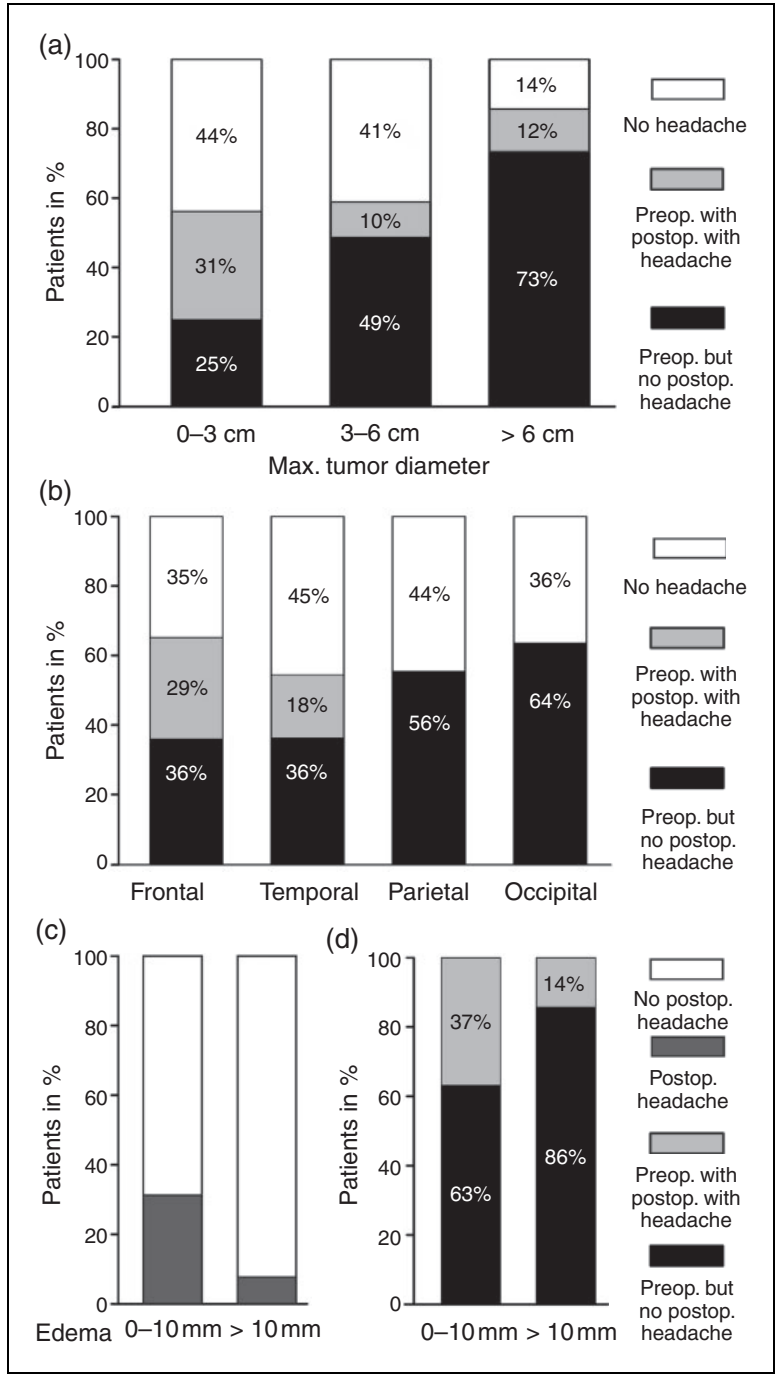

Figure 3. Postoperative headache. (a) Patients with high tumor progression had a large reduction in headache due to neurosurgical intervention. (b) A complete postoperative absence of headache was observed for parietal and occipital localized meningiomas, compared to a reduction in frontal and temporal cases. (c) Percentage of patients with and without postoperative headache separated by preoperative edema. (d) Within the subgroup of patients with preoperative headache, the percentage of patients with and without postoperative headache separated by preoperative edema. The percentage of patients is given within the bars of the stacked histograms.

In patients with frontal and temporal meningioma, the postoperative incidence of headache was about half of the preoperative incidence; for the parietal and occipital cases, all patients were headache-free after the operation (Figure 3(b)). Anatomical locations of particular interest did not comprise enough patients to allow meaningful statistical analysis. In 57 of 69 patients, the meningioma could be fully removed. Complete vs. incomplete removal was not predictive for occurrence of postoperative headache ( $p=0.86$, Chi-square test). Patients with a preoperative edema above $10 \mathrm{~mm}$ reported a postoperative headache in $8 \%(2 / 26)$, which is lower than the $31 \%(10 / 32)$ of patients reporting headache with smaller preoperative edema $(p=0.028$, Chisquare test, Figure 3(c)).

According to the study protocol, the three patients with intracranial pathology were not excluded. The supplementary statistics tables provide all results presented above also as sub-analysis for the 66 patients without intracranial pathology, which did not show substantial differences.

\section{Discussion}

We investigated patients' headache together with structural, biochemical and histochemical tumor parameters before and after meningioma surgery. Edema diameter and malignancy index MIB-1 were negatively correlated with preoperative headache occurrence, and a large tumor size was the most important parameter for a high chance of a postoperative headache reduction. In our patient sample, a high level of tumor progression favors intervention and in these patients the biggest improvements in tumor-associated headache are predicted.

It should be noted that the study investigated a preoperative patient cohort seen and selected for neurosurgery at the Department of Neurosurgery of the University of Erlangen-Nürnberg. This might implicate a selection bias compared to a cohort of patients with meningioma at the stage of first diagnosis. For example, the percentage of patients with symptoms due to the meningioma is likely to be enriched in a preoperative cohort referred to neurosurgery when compared to all subjects with a meningioma, of which many are asymptomatic or not diagnosed. On the other hand, only one migraine patient was in our sample, substantially below an expected 6.2 of 69 patients with migraine, calculated on the basis of age-matched prevalence (35). We cannot exclude undiagnosed primary headaches in the present study. However, most primary headaches occur earlier in life and are therefore typically known already at the time of meningioma incidence. In primary headache patients, referral to structural diagnostics might have a higher threshold. Further, even for those who received structural diagnostics, there might be a bias against referral to neurosurgery. Together both reasons might explain why primary headaches did occur less frequently in our patients than expected from an unbiased sample.

There was a substantial positive correlation between the tumor size and the edema diameter, which is not surprising. However, tumor size but not edema diameter was a good predictor for postoperative outcome. Although there are many studies about brain tumor 
headache and possible involvement of diverse substances which might be released, there are still open questions that have to be answered. There is seminal work regarding the pain-sensitive structures in the meninges, which are perceived as localized headache according to the site of stimulation $(36,37)$. They showed that the dura mater is sensitive to stimulation, and pain is in particular observed when stimulation was performed close to arterial vessels, where the branches of the trigeminal nerve travel alongside.

Pre-existing primary headache is a risk factor for developing headache from a brain tumor, which suggests a predisposition for secondary headache (4). In more than $80 \%$ of the patients, the headache changed with the occurrence of the tumor. Stimulation of the trigeminal ganglion led to an increase in regional cerebral blood flow in the frontal and parietal cortex of cats (38). The particular importance of the neuropeptide CGRP in meningeal nociception was the topic of several investigations (39-41). Headaches associated with brain tumors were commonly assumed to be the result of intracranial pressure and mechanical traction on pain-sensitive structures. In addition to the mechanical activation suggested by the results of Ray and Wolff (37), there are likely also chemical pronociceptive effects of tumors affecting the nociceptive afferents. For patients with brain tumors of all etiologies, the incidence of headache was about $50 \%$ (42).

For our analysis, meningiomas were subdivided into different innervation territories of the three trigeminal branches and the cervical spinal nerves C1-C3. Meningiomas in the area of the ophthalmic branch were more frequently associated with preoperative headache compared to locations associated with other branches of the trigeminal nerve. The innervation of the ophthalmic branch includes the supraorbital region. It was described that periorbital allodynia occurs due to an injury in the somatosensory cortex (43). There is convergent evidence that chemical sensitization or activation of nociceptive afferents innervating the dura mater can cause facial allodynia $(44,45)$. The most likely explanation is convergent input to central nociceptive structures, because the ophthalmic branch of the trigeminal nerve innervates most of the facial areas and the dura mater (46). Furthermore, the involvement of the cavernous sinus has been considered as a source of headache, since several structures involved in headache generation such as the internal carotid artery and the ophthalmic branch of the trigeminal nerve pass here, but this remained rather speculative (47). Dural stretch was a traditional explanation for the occurrence of headache, which has not been substantiated.

Our findings show a negative correlation between preoperative edema diameter and preoperative headache rating of the patients. This appears counterintuitive at first glance, as a larger edema diameter might cause more intracranial pressure and headache. It should be considered that pressure at nerves could cause a conduction block and thereby suppress or silence neurons. An important aspect is the tumor growth rate. Fast growing tumors are more rapidly detected than slowly growing ones. The faster growing tumors, also associated with more edema, might dislocate other structures more effectively compared to the slower growing tumors, which may invade structures without displacing them. Furthermore, the tumor subtype might be important, and there might be pronociceptive paraneoplastic effects of particular tumor types.

Patients without preoperative headache had a higher tumor proliferation index (MIB-1). As mentioned above, faster growing tumors seem to be detected by other symptoms than pain. The concentration of $\mathrm{PGE}_{2}$ in the meningioma was weakly positively associated with preoperative headache. $\mathrm{PGE}_{2}$ is a well-known sensitizer of nociceptive molecular targets such as TRPV1 (49), which could further promote pronociceptive cascades, including neurogenic inflammation and activation of non-neuronal structures. Inflammatory reactions caused by the tumor or brain injuries can induce plasma extravasation and extracellular accumulation of fluid and proteins. A dysfunction of tightjunction proteins may also play a role in the occurrence of edema. Inflammation can also be associated with an increased permeability of the blood brain barrier, which might increase edema, and which might expose nerve fibers to additional excitatory substances.

Fortunately, a large fraction of patients is headachefree after resection. This occurs more frequently in patients with larger tumors compared to smaller tumors. The relief of headache in particular in patients with larger tumors could be explained by the mechanical effects of the larger volume removed, but other parameters associated with size such as growth rate and malignancy should also be considered. Associated with the latter factors are also biochemical changes, particularly paracrine (paraneoplastic) effects of the tumor on its surroundings, which could contribute to secondary headache.

Considering the tumor location, patients with preoperative headache and tumors with parietal or occipital location were completely headache-free after surgery. An explanation might be the available space, which might be more tightly limited in areas where expansion might affect the brainstem. Still, more than half of the patients with temporal and frontal tumors became headache-free after surgery. By trend, patients with a large preoperative edema had more relief of headache after resection of their tumor, which again could be explained by the mechanical alteration as well as biochemical changes associated with the edema. In males, 
the WHO grade was higher, and tumor diameter and edema larger compared to females. This could be explained by more aggressive tumors in males, but more likely by a well-described higher level of indolence in males, seeking medical attention in a later stage. A final point to be considered is whether neurons grow into tumors due to chemo-attractive stimuli or are changed in their peripheral expression. Larger tumors had a higher substance P concentration. Substance P levels were most substantial in tumors located in the frontal lobes, but this might at least partially be explained by the meningeal innervation pattern.

To specifically address a possible involvement of CGRP, substance $\mathrm{P}$ and $\mathrm{PGE}_{2}$ in preoperative headache with adjustment for confounders, we used multivariable regression models. The intake of pain medication and tumor size were regarded as potential confounders; thus, they were added in a first regression model (Model 1). Adding CGRP, substance $\mathrm{P}$ and $\mathrm{PGE}_{2}$ in addition (Model 2) allowed testing for if and which of these variables have additional predictive value. First, Firth logistic regression was used to model the occurrence (rating 0 vs. $>0$ ) of preoperative headache. The fact that pain medication significantly predicted the presence of preoperative headache is not surprising. The addition of CGRP, substance $P$ and $\mathrm{PGE}_{2}$ concentration did not improve the predictive value of the model, indicating that there is no evidence that these molecules are involved in the occurrence of preoperative headache. Importantly, due to the limited statistical power of this exploratory trial, this cannot be interpreted as no involvement, it merely indicates the absence of evidence for an involvement.

Regarding pain intensity (rating 1-51) in patients with preoperative headache, however, multiple linear regression supports an involvement of substance $\mathrm{P}$ and $\mathrm{PGE}_{2}$. Together with CGRP concentration, the concentration of these two molecules within meningiomas explained considerably more variance of preoperative headache intensity than pain medication and tumor size alone. The results suggest that doubling of substance $P$ and $\mathrm{PGE}_{2}$ concentration increase headache intensity by 4.4 and 2.5 units. The non-significant effect of CGRP concentration means that there could be an association with preoperative headache, but our data provide no evidence for it. Significant overall likelihood ratio chi-square statistics suggest that pain medication, maximal tumor diameter, substance $\mathrm{P}$ and $\mathrm{PGE}_{2}$ predict preoperative headache generally.

Limitations of the study: The chosen questionnaire has some disadvantages. This includes the pain rating scale, which uses a verbal grouping that might affect the linearity of the scale, a non-standard number range, and an untypical upper end of the scale. Further, the questionnaire was not specifically designed for the present study, and therefore an unnecessary effort was required to answer questions that were not of interest for this study. Patients were included in the neurosurgery department, therefore at a stage when a surgical option was at least seriously considered. This limits generalization to other patient cohorts, for example at an earlier stage in the clinical decision tree. Based on the late inclusion, the study lacks presurgical data such as headache frequency and intensity based on a headache diary. It should be considered that dexamethasone application before surgery might have had an influence on the levels of the biomarkers. Postoperative headaches were investigated once, one year after surgery; no time course of headache incidence was acquired. The limited sample size did not allow for statistically significant numbers in a finer categorization and might have overlooked existing associations due to the generalization required to get sufficient numbers per group. A placebo rate due to surgical procedures should be considered when evaluating the results (50), especially taking into account that more invasive procedures have higher placebo rates.

With special focus on associated headache, several new associations between clinical and experimental measures have been found in operated patients with meningioma. However, it has to be noted that due to the exploratory character of this study, these results need to be interpreted with caution and considered to be new hypotheses. Furthermore, due to the observational study design, no causal relationships can be established. In this regard, a causal involvement of substance $\mathrm{P}$ might be probed in a clinical study testing the efficacy of the approved NK1-receptor antagonist aprepitant as a symptomatic therapy against tumor-induced headache.

\section{Key findings}

- Preoperative headache intensity was associated with the innervating trigeminal branch, the surrounding edema, tumor proliferation index MIB-1 and with prostaglandin $\mathrm{E}_{2}$ content. After adjusting for pain medication intake and maximal tumor diameter, an increase of substance $\mathrm{P}$ and of $\mathrm{PGE}_{2}$ concentration within tumors was associated with higher preoperative pain intensity.

- A high maximal tumor diameter was a predictor for a low probability of postoperative headache 


\section{Acknowledgements}

We like to thank Birgit Vogler, Annette Kuhn and Susanne Haux-Örtel for excellent technical assistance. We thank Rok Blagus and Georg Heinze (Medical University of Vienna, Institute of Clinical Biometrics) for statistical advice with regards to multivariable analyses. Author contributions: FG designed the study, acquired patient data, performed experiments and drafted the manuscript. FS designed the study, acquired material and interpreted patient data. $\mathrm{SH}$ performed multivariable analyses and wrote respective parts of the manuscript. MB contributed to the manuscript. KM coordinated the study and wrote the manuscript. MJMF supported experiments, performed statistical analysis, compiled the graphical presentation and wrote the manuscript.

\section{Declaration of conflicting interests}

The authors declared no potential conflicts of interest with respect to the research, authorship, and/or publication of this article.

\section{Funding}

The authors received no financial support for the research, authorship, and/or publication of this article.

\section{ORCID iD}

Michael JM Fischer (D) http://orcid.org/0000-0002-3811-7066

\section{References}

1. Gelabert-González M, Fernández-Villa JM and IglesiasPais M. Atypical and malignant intracranial meningiomas. Rev Neurol 2004; 38: 304-310.

2. Gelabert-González M, García-Allut A, Bandín-Diéguez J, et al. Meningiomas of the lateral ventricles. A review of 10 cases. Neurocir Astur Spain 2008; 19: 427-433.

3. Moradi A, Semnani V, Djam H, et al. Pathodiagnostic parameters for meningioma grading. J Clin Neurosci 2008; 15: 1370-1375.

4. Schankin CJ, Ferrari U, Reinisch VM, et al. Characteristics of brain tumour-associated headache. Cephalalgia 2007; 27: 904-911.

5. Levy MJ, Matharu M and Goadsby PJ. Chronic headache and pituitary tumors. Curr Pain Headache Rep 2008; 12: 74-78.

6. Levy MJ, Classey JD, Maneesri S, et al. The association between calcitonin gene-related peptide (CGRP), substance $\mathrm{P}$ and headache in pituitary tumours. Pituitary 2004; 7: 67-71.

7. Schankin CJ, Krumbholz M, Sostak P, et al. Headache in patients with a meningioma correlates with a bone-invasive growth pattern but not with cytokine expression. Cephalalgia 2010; 30: 413-424.

8. Broholm H, Rubin I, Kruse A, et al. Nitric oxide synthase expression and enzymatic activity in human brain tumors. Clin Neuropathol 2003; 22: 273-281.

9. St-Jacques B and Ma W. Peripheral prostaglandin E2 prolongs the sensitization of nociceptive dorsal root ganglion neurons possibly by facilitating the synthesis and anterograde axonal trafficking of EP4 receptors. Exp Neurol 2014; 261: 354-366.

10. Harvey RJ, Depner UB, Wässle H, et al. GlyR alpha3: An essential target for spinal PGE2-mediated inflammatory pain sensitization. Science 2004; 304: 884-887.

11. Lin C-CD, Kenyon L, Hyslop T, et al. Cyclooxygenase-2 (COX-2) expression in human meningioma as a function of tumor grade. Am J Clin Oncol 2003; 26: S98-S102.

12. Oikonomou E, Machado AL, Buchfelder $\mathrm{M}$, et al. Meningiomas expressing and responding to cholecystokinin (CCK). J Neurooncol 2005; 73: 199-204.

13. Biroli A and Talacchi A. Surgical management of lateral tentorial meningiomas. World Neurosurg 2016; 90: 430-439.

14. Bumrungrachpukdee P, Pruphetkaew N, Phukaoloun M, et al. Recurrence of intracranial meningioma after surgery: Analysis of influencing factors and outcome. J Med Assoc Thail Chotmaihet Thangphaet 2014; 97: 399-406.

15. Di Cristofori A, Zarino B, Bertani G, et al. Surgery in elderly patients with intracranial meningioma: Neuropsychological functioning during a long term follow-up. J Neurooncol 2018; 137: 611-619.

16. Maiti TK, Bir SC, Patra DP, et al. Spinal meningiomas: Clinicoradiological factors predicting recurrence and functional outcome. Neurosurg Focus 2016; 41: E6; doi: 10.3171/2016.5.FOCUS16163.

17. Mehdorn HM. Intracranial meningiomas: A 30-year experience and literature review. Adv Tech Stand Neurosurg 2016; 43: 139-184.

18. Murrone D, De Paulis D, di Norcia V, et al. Surgical management of falcine meningiomas: Experience of 95 patients. $J$ Clin Neurosci 2017; 37: 25-30.

19. Ottenhausen M, Ntoulias G, Bodhinayake I, et al. Intradural spinal tumors in adults - update on management and outcome. Neurosurg Rev. Epub ahead of print 17 February 2018. DOI: 10.1007/s10143-018-0957-x).

20. Poon MT-C, Fung LH-K, Pu JK-S, et al. Outcome of elderly patients undergoing intracranial meningioma resection - a systematic review and meta-analysis. $\mathrm{Br} \mathrm{J}$ Neurosurg 2014; 28: 303-309.

21. Riad H, Knafo S, Segnarbieux F, et al. Spinal meningiomas: Surgical outcome and literature review. Neurochirurgie 2013; 59: 30-34.

22. Shin M, Kondo K and Saito N. Current status of endoscopic endonasal surgery for skull base meningiomas: Review of the literature. Neurol Med Chir (Tokyo) 2015; 55: 735-743.

23. Wu A, Garcia MA, Magill ST, et al. Presenting symptoms and prognostic factors for symptomatic outcomes following resection of meningioma. World Neurosurg 2018; 111: e149-e159.

24. Wu Z, Hao S, Zhang J, et al. Foramen magnum meningiomas: Experiences in 114 patients at a single institute over 15 years. Surg Neurol 2009; 72: 376-382; discussion 382.

25. Headache Classification Committee of the International Headache Society (IHS). The International Classification of Headache Disorders, 3rd edition (beta version). Cephalalgia 2013; 33: 629-808. 
26. Fischer MJM and Reeh PW. Sensitization to heat through G-protein-coupled receptor pathways in the isolated sciatic mouse nerve. Eur J Neurosci 2007; 25: 3570-3575.

27. Bretag AH. Synthetic interstitial fluid for isolated mammalian tissue. Life Sci 1969; 8: 319-329.

28. Ebersberger A, Averbeck B, Messlinger K, et al. Release of substance $\mathrm{P}$, calcitonin gene-related peptide and prostaglandin E2 from rat dura mater encephali following electrical and chemical stimulation in vitro. Neuroscience 1999; 89: 901-907.

29. Abramovich CM and Prayson RA. MIB-1 labeling indices in benign, aggressive, and malignant meningiomas: A study of 90 tumors. Hum Pathol 1998; 29: 1420-1427.

30. Scholzen $\mathrm{T}$ and Gerdes J. The Ki-67 protein: From the known and the unknown. J Cell Physiol 2000; 182: 311-322.

31. Firth D. Bias reduction of maximum likelihood estimates. Biometrika 1993; 80: 27-38.

32. Heinze $G$ and Schemper M. A solution to the problem of separation in logistic regression. Stat Med 2002; 21: 2409-2419.

33. Kirby $\mathrm{S}$ and Purdy RA. Headaches and brain tumors. Neurol Clin 2014; 32: 423-432.

34. Wirth FP and Van Buren JM. Referral of pain from dural stimulation in man. J Neurosurg 1971; 34: 630-642.

35. Buse DC, Loder EW, Gorman JA, et al. Sex differences in the prevalence, symptoms, and associated features of migraine, probable migraine and other severe headache: Results of the American Migraine Prevalence and Prevention (AMPP) Study. Headache 2013; 53: 1278-1299.

36. Feindel W, Penfield W and McNaughton F. The tentorial nerves and Iocalization of intracranial pain in man. Neurology 1960; 10: 555-563.

37. Ray BS and Wolff HG. Experimental studies on headache: Pain-sensitive structures of the head and their significance in headache. Arch Surg 1940; 41: 813-856.

38. Goadsby PJ and Duckworth JW. Effect of stimulation of trigeminal ganglion on regional cerebral blood flow in cats. Am J Physiol 1987; 253: R270-R274.

39. Fischer MJM, Koulchitsky $\mathrm{S}$ and Messlinger $\mathrm{K}$. The nonpeptide calcitonin gene-related peptide receptor antagonist BIBN4096BS lowers the activity of neurons with meningeal input in the rat spinal trigeminal nucleus. $J$ Neurosci 2005; 25: 5877-5883.

40. Sixt M-L, Messlinger K and Fischer MJM. Calcitonin gene-related peptide receptor antagonist olcegepant acts in the spinal trigeminal nucleus. Brain J Neurol 2009; 132: 3134-3141.

41. Storer RJ, Akerman S and Goadsby PJ. Calcitonin generelated peptide (CGRP) modulates nociceptive trigeminovascular transmission in the cat. $B r J$ Pharmacol 2004; 142: 1171-1181.

42. Forsyth PA and Posner JB. Headaches in patients with brain tumors: A study of 111 patients. Neurology 1993; 43: 1678-1683.

43. Elliott MB, Oshinsky ML, Amenta PS, et al. Nociceptive neuropeptide increases and periorbital allodynia in a model of traumatic brain injury. Headache 2012; 52: 966-984.

44. Burstein R, Yamamura H, Malick A, et al. Chemical stimulation of the intracranial dura induces enhanced responses to facial stimulation in brain stem trigeminal neurons. J Neurophysiol 1998; 79: 964-982.

45. Wieseler J, Ellis A, Sprunger D, et al. A novel method for modeling facial allodynia associated with migraine in awake and freely moving rats. $J$ Neurosci Methods 2010; 185: 236-245.

46. Shankland WE. The trigeminal nerve. Part II: The ophthalmic division. Cranio J Craniomandib Pract 2001; 19: $8-12$.

47. Sjaastad O. Cluster headache: The possible significance of midline structures. Cephalalgia 1988; 8: 229-236.

48. Levy MJ. The association of pituitary tumors and headache. Curr Neurol Neurosci Rep 2011; 11: 164-170.

49. Ma W, St-Jacques B, Rudakou U, et al. Stimulating TRPV1 externalization and synthesis in dorsal root ganglion neurons contributes to PGE2 potentiation of TRPV1 activity and nociceptor sensitization. Eur J Pain Lond Engl 2017; 21: 575-593.

50. Gu AP, Gu CN, Ahmed AT, et al. Sham surgical procedures for pain intervention result in significant improvements in pain: Systematic review and meta-analysis. J Clin Epidemiol 2017; 83: 18-23. 